OPEN ACCESS

Edited by:

Taiki Takahashi,

Hokkaido University, Japan

Reviewed by:

Sergio Da Silva,

Universidade Federal de Santa

Catarina, Brazi

Barret Pengyuan Shao,

Independent Researcher,

United States

${ }^{*}$ Correspondence:

Arthur E. Attema

attema@eshpm.eur.n!

Specialty section:

This article was submitted to

Mathematical Finance,

a section of the journal

Frontiers in Applied Mathematics and

Statistics

Received: 06 March 2018 Accepted: 02 May 2018

Published: 23 May 2018

Citation:

Attema AE and Lipman SA (2018)

Decreasing Impatience for Health

Outcomes and Its Relation With

Healthy Behavior.

Front. Appl. Math. Stat. 4:16. doi: 10.3389/fams.2018.00016

\section{Decreasing Impatience for Health Outcomes and Its Relation With Healthy Behavior}

\author{
Arthur E. Attema* and Stefan A. Lipman \\ Erasmus School of Health Policy \& Management, Erasmus University Rotterdam, Rotterdam, Netherlands
}

There is a growing amount of literature suggesting people tend to behave inconsistently over time, which is driven by decreasing impatience. In addition, many studies have found relations between discounting estimates from experiments and field behavior, such as smoking cessation and dieting. However, these studies often did not separate time inconsistency from other factors such as utility curvature or the level of discounting. In order to establish the relation between field behavior and the degree of time inconsistency, it is therefore necessary to obtain a pure measure of the latter that is not distorted by these other factors. The present study implements a recently introduced measure of deviations from constant impatience, called the "Decreasing Impatience (DI)-index," to estimate the degree to which people deviate from constant impatience. We provide the first extension of DI to health outcomes, both for individual and societal discounting using three different starting points. Moreover, we include a survey gathering information about several health-related behaviors, in order to test for the relationship between the amount of decreasing impatience and healthy behavior. We observe that decreasing impatience is the modal preference, although constant and increasing impatience are no exceptions, and, hence, these types of discounters should not be neglected. Furthermore, the DI-index is higher for individual health outcomes than for societal health outcomes, but is not distributed differently among the three classes of discounters. The Dl-index decreases with starting period for individual health outcomes, but not for societal health outcomes. Very few significant relations between time inconsistency and self-reported health-related behavior were found.

Keywords: decreasing impatience, health, increasing impatience, time inconsistency, time preference

\section{INTRODUCTION}

Many daily decisions require an intertemporal trade-off between earlier and later consequences. These vary from savings for pensions, to learning for exams, to more exercise now to reduce the chance of becoming obese later. In these decisions, agents' discount rates play an important role. Economic theory predicts that the more agents discount the future, the less they will engage in future-oriented behavior, such as saving. During the last few decades it has become clear that besides the discount rate, the amount of time inconsistency is also highly relevant for many decisions. For example, heterogeneity in time inconsistency may explain why agents with the same absolute discount rate differ in their tendency to postpone an annoying task [1]. 
Because of their differential impact on intertemporal choices, it is crucial to disentangle time inconsistency and discount rates in empirical studies. Furthermore, these two factors may both be confused with utility curvature, which also affects most elicitations of discounting parameters [2, 3]. A recent study proposed a way to disentangle these three fundamentally different concepts, including a first empirical test demonstrating its feasibility [1]. In particular, Rohde [1] introduced the Decreasing Impatience (DI)-Index, which is a summary measure of the degree to which an agent deviates from constant discounting; i.e., the degree to which the agent is time inconsistent. She showed that this measure is neither affected by the level of impatience, nor by the shape of the agent's utility function. The experimental results reported in her paper indicated that, for monetary outcomes, decreasing impatience was the modal type of discounting, confirming most of the previous literature. However, it also became clear that a sizable minority of subjects was increasingly impatient, highlighting the need to account for this type of preferences as well. Finally, Rohde's [1] experiment was complemented by a survey asking several questions about health-related behavior and found no significant associations between those and the DI-index.

This lack of association is surprising, given the perceived importance of decreasing impatience, and may have several explanations. One of them is simply a lack of power, but another one may be the use of money as a stimulus used in the elicitation of the DI-index, to predict health-related behavior. Earlier work, however, has demonstrated that deviations from constant discounting are more pronounced for health outcomes compared to monetary outcomes [4]. As such, the lack of association reported in Rohde [1] may be explained by the disparity between elicitation and outcome. To test this hypothesis, we elicit four DIindices using health outcomes in this study: two in an individual context and two in a societal context. In addition, we implement the same survey as Rohde to see if the use of health outcomes allows us to observe a significant association between time consistency and health behaviors, such as smoking and alcohol consumption.

This paper is organized as follows. We introduce the theoretical background in section Theoretical background. After that, we describe the experimental design in section Experiment, followed by the results in section Results. Finally, in section Discussion, we discuss the results, and conclude.

\section{THEORETICAL BACKGROUND}

\section{Notation}

In our experiment, we will consider indifferences between timed outcomes ( $\mathrm{t}: \mathrm{x}$ ), where $\mathrm{x}$ denotes a health improvement and $\mathrm{t}$ denotes its time of onset. We consider the usual preference relation $\succcurlyeq$ over these outcomes. A weak [strict] preference is denoted by $\succcurlyeq[\succ]$ and indifference by $\sim$. Throughout the paper

Abbreviations: DBI, Duration before implementation; DI, Decreasing impatience; DU, Discounted utility; EQ-5D, EuroQol Five-Dimension; ERIM, Erasmus Research Institute of Management. we assume the discounted utility (DU) model to hold:

$$
D U(x, t)=\delta(t) U(x)
$$

Here, DU denotes discounted utility, $\delta(t)$ is the discount function, and $\mathrm{U}(\mathrm{x})$ is the instantaneous utility of outcome $\mathrm{x}$. The standard DU model is the constant discounting model [5], which models intertemporal outcome profiles by the following formula:

$$
D U\left(x_{t}, x_{t+1}, \ldots, x_{T}\right)=\sum_{t=0}^{T} \gamma^{t} U\left(x_{t}\right),
$$

where $\gamma$ represents the discount factor. One of the axioms of this model is stationarity, which causes agents to always act time consistently; i.e., they stick to their plans [6]. However, many empirical studies have demonstrated that agents often will not behave this way, with a tendency to postpone annoying tasks (e.g., doing homework, stopping smoking) and to indulge in activities giving immediate benefits (e.g., purchasing a car, eating candy) $[7,8]$. This kind of behavior can often be explained by hyperbolic discounting models that incorporate decreasing impatience. The most popular hyperbolic discounting function is quasi-hyperbolic discounting $[9,10]$ :

$$
D U\left(x_{t}, x_{t+1}, \ldots, x_{T}\right)=U\left(x_{0}\right)+\sum_{t=1}^{T} \beta \delta^{t} U\left(x_{t}\right) .
$$

Here, $0 \leq \beta \leq 1$ represents a measure of the "immediacy effect" or "present bias," giving a penalty to all outcomes occurring in the future, but not discriminating between the amounts of the delay for $t>0$. This model reduces to constant discounting for the special case where $\beta=1$. In other words, the quasi-hyperbolic model assumes that agents are decreasingly impatient only when the present is involved, and constantly impatient when only comparing future outcomes. By contrast, alternative models allow for universally decreasing impatience, i.e., even if the present is not involved (e.g., power discounting [11], proportional discounting [12], generalized hyperbolic discounting [13], and nonlinear time perception [14]).

\section{Related Literature}

Some previous studies have investigated the measurement of decreasing impatience. First, Prelec [15] proposed to assess the degree of Pratt-Arrow convexity of the logarithm of the discount function. However, this method is hard to implement in practice because it requires assumptions about or measurement of the utility function, and also specifying a parametric form of the discount function, for example assuming constant discounting. There is overwhelming empirical evidence of violations of constant discounting in the monetary domain [16], but more recently such violations have also been widely documented for health outcomes [17, 18]. In both domains, there is increasing evidence that a substantial minority of subjects is increasingly impatient, both for money [19] and for health [4], highlighting the need to accommodate this behavior as well [20]. However, to the best of our knowledge, no studies have been performed yet that measure the degree of time 
inconsistency for health outcomes, be it decreasing or increasing impatience.

As can be seen from Equation 1, intertemporal choices are not only affected by time preferences, but also by utility curvature. Therefore, when attempting to measure discount functions, it is important to assure they are separated from any effects of utility on intertemporal choices. Rohde [21] developed the hyperbolic factor to simplify this practical implementation, which was measured by Attema et al. [19], who found that a majority of choices satisfied increasing impatience. A few years later, Bleichrodt et al. [4] used the method of Attema et al. in the health domain, where they observed decreasing impatience to be the dominant pattern, but also a substantial minority who were increasingly impatient. However, as explained by Rohde [1], the hyperbolic factor can only be computed for modest amounts of decreasing and increasing impatience. This led her to propose the DI-index, which does not suffer from this drawback, and she measured it for monetary outcomes. Our study is the first to measure the DI-indices for health outcomes. As such, in this study we are able to measure the degree of time inconsistency without any distortion caused by utility curvature.

\section{EXPERIMENT}

\section{Subjects and Design}

Our subject pool consisted of a sample of 99 university students (47 female, 50 male, 2 unknown), with a mean age of 19.3 $(\mathrm{SD}=1.6)$. Subjects were recruited by the Erasmus Research Participation System and the experiment was administered in the Erasmus Behavioral Lab. The subjects received course credits for their participation. This study was carried out in accordance with the recommendations of the Erasmus Research Institute of Management (ERIM) Internal Review Board. The protocol was approved by the ERIM Internal Review Board, Section Experiments. All subjects gave written informed consent in accordance with the Declaration of Helsinki. This study used a within-subjects design, to determine DI-indices for both individual and societal decision-making for health outcomes. All subjects completed the individual task first, after which they completed the societal task. Our stimuli-durations for the both tasks were chosen to maximize comparability to Rohde's [1] study. Hence, we set these durations to 0,2 , and 4 months, with these stimuli-durations being presented in increasing order in the survey.

\section{Procedure}

Subjects received paper-and-pencil instructions (see Appendix A) for this study as part of a larger survey on health-related decision-making, which is not discussed in this paper. For the individual task, subjects had to assume they were experiencing chronic back pain, as described by the following problems:

- You have moderate problems in walking about.

- You have moderate problems performing your usual activities (e.g., work, study, housework, family or leisure activities).

- You have moderate pain or discomfort.
Subjects were instructed that two treatments (A and B) were available to relieve this chronic back pain. The health improvements of treatment $\mathrm{A}$ and $\mathrm{B}$ were based on the description suggested by Bleichrodt et al. [4], and consisted of an improvement in some dimensions of the EuroQol FiveDimension (EQ-5D) classification system. The descriptions of these two treatments were presented to subjects on a separate paper, which was put on subjects' desks. In all cases, Treatment B was more effective than Treatment A. Both treatments removed the pain, but $B$ also improved the problems with walking and usual activities. The effects of the treatments started immediately at its onset and lasted for exactly one week. The amount of time before the treatments occur will be referred to as "duration before implementation" (DBI). In all cases, chronic back pain would return after 1 week. Such questions are common in elicitations of time preferences for health outcomes, except that usually only one change in health is studied (e.g., Treatment A), with its duration being varied [22, 23]. As explained by Bleichrodt et al. [4], the advantages of keeping the duration of change fixed are that the utility for time duration can be entirely general, without having to impose simplifying assumptions to enable the analysis of responses, and that subjects will more likely concentrate on the time point at which the change occurs (i.e., the DBI). The latter is desirable if one is interested in the properties of the discount function and not in those of the utility function.

The societal task used a similar set-up, except that in this task the health improvements did not accrue to the subjects personally but to a group of students (similar to them), who were suffering from chronic back pain. The description of this chronic health state was identical to the description in the individual task. Subjects had to trade off a higher amount of people receiving the same health improvement to a delay of the realization of this health improvement. More specifically, they faced a tradeoff between treating 40 students (Option A) or incurring a delay to treat 50 students (Option B). These numbers were also used by Rohde [1], in terms of monetary outcomes (i.e., $€ 40$ and $€ 50$ ). By using these numbers, our ratio of the earlier to the later outcomes was the same, facilitating comparison of the discount rates. The treatment was the same in both conditions (individual vs. societal), being Treatment B of the individual task, i.e., alleviating the pain and problems on other dimensions for 1 week.

In both the societal and individual task, subjects faced a choice list (see Appendix A for an example), with Treatment A on the left hand side, given a fixed DBI $\left(t_{0-2}\right)$, and Treatment B on the right hand side with a monotonically increasing DBI. We elicit indifferences at $s_{0}=0, s_{1}=2$ months, and $s_{2}=4$ months. These numbers were also used by Rohde [1], except that she used weeks instead of months. We did so because our health improvement lasted 1 week and it might have caused confusion if both this duration and the delay were expressed in months. Before subjects started working on these choice lists, they faced several questions aimed at determining comprehension. We infer indifference at the DBI where subjects switch from B to A, in agreement with the conventional use of choice lists in experimental economics [24]. 


\section{Calculation of DI-Indices}

Summarizing, we obtain three indifferences for both individual and societal health outcomes. Indifferences of the form $\left(s_{i}: x\right) \sim$ $\left(t_{i}: y\right)$ can be evaluated by the following equation under DU (Equation 1):

$$
\mathrm{D}\left(s_{i}\right) \mathrm{U}(\mathrm{x})=\mathrm{D}\left(t_{i}\right) \mathrm{U}(\mathrm{y}) \text { with } \mathrm{i}=0,1 \text { or } 2 .
$$

As is described by Rohde [1], the DI-index can be computed from every two indifferences of the form $(\mathrm{s}: \mathrm{x}) \sim(\mathrm{t}: \mathrm{y})$ and $(\mathrm{s}+\sigma: \mathrm{x}) \sim(\mathrm{t}+\tau$ :y) [1]. In our experiment we obtain $\mathrm{s}, \mathrm{t}, \tau$ and $\sigma$ as follows: for any $s_{i}$, with $i=0,1$ or 2 , we set $s_{i}=s$. We then obtain $\mathrm{t}$ by the elicited indifference $\left(s_{i}: x\right) \sim\left(t_{i}: y\right)$, with $\mathrm{t}=t_{i}$. Next, we set $\sigma$, which corresponds here to setting $\sigma=s_{i+1}-s_{i}$, and elicit the indifference $\left(s_{i+1}: x\right) \sim\left(t_{i+1}: y\right)$. We proceed by finding $\tau$ by determining $t_{i+1}-t_{i}$. The DI-index is then given by:

$$
D I=\frac{\tau-\sigma}{\sigma(t-s)}
$$

where (as shown by Rohde [1]) constant [decreasing, increasing] impatience corresponds to a DI-index of $0[>0,<0]$.

In the case of societal discounting, we elicit similar indifferences to $\left(s_{i}: x\right) \sim\left(t_{i}: y\right)$ where $\mathrm{x}$ and $\mathrm{y}$ are replaced by $m$ and $n$, where $m$ and $n$ are the number of patients treated at time period, e.g., $\left(s_{i}: 40\right) \sim\left(t_{i}: 50\right)$. The derivation of DI-indices does not change.

Because two questions are needed to elicit one DI-index, our design enabled us to elicit two DI-indices for both the individual and the societal task, where we will indicate individual DI-indices by DI-I and societal DI-indices by DI-S. We furthermore add to these the pre-set durations used to derive them, yielding DI-I-02 and DI-I-24 (and DI-S-02 and DI-S-24 for societal).

To give an example, imagine that for the individual task a subject in our study has the following indifferences:

$$
\begin{aligned}
& (0: x) \sim(5: y) \\
& (2: x) \sim(8: y) \\
& (4: x) \sim(13: y)
\end{aligned}
$$

This means that we have $s=0$ and $\sigma=2$ for the first two indifferences, and $s=2$ and $\sigma=2$ for the last two indifferences. We elicited indifferences at t's $=5,8,13$. This gives $\mathrm{t}=5$ and $\tau=3$ when the DI-index is calculated based on the first two indifferences, yielding DI-I-02 $=0.1$. Similarly, when constructing the DI-index of the second and third indifferences, this yields DI-I-24 $=0.25$. Hence, this subject would be classified as decreasingly impatient.

\section{Survey Questions}

In order to maximize comparability with the study of Rohde [1], we implemented the same questions in an accompanying survey. This consisted of a number of demographic and behavioral characteristics and the self-control questions of Ameriks et al. [25]. The survey developed by Ameriks et al. [25] aims to measure self-control problems with a self-reported questionnaire. Additional self-awareness questions concerning sports, study, and class preparation were asked [1, 26], which were administered on an 8-point Likert scale. In this paper we focus on the role of DI-indices in health behavior. As such, our results for self-control problems and self-awareness can be found in Appendix B. The health behavior variables measured [using identical questions as in Rohde [1]] include the number of hours of sports per week, smoking behavior, amount of alcohol consumption per week, and length and weight (out of which the body mass index was computed). The following demographics were obtained: age, gender, whether or not respondents live with their parents, nationality (Dutch or non-Dutch), and whether and how much money they saved. In addition, we measured subjects' health status on a 10-point scale and their subjective life expectancy.

\section{RESULTS}

Five subjects did not complete the societal task, while one did not complete the individual task. As in Rohde [1], the number of subjects who always chose the patient option was quite high, especially for the societal task (12 never switched in both tasks, and 26 subjects did not switch in the societal task). These subjects had to be excluded from the analysis of decreasing impatience, since it was not possible to compute a DI-index for them (14 subjects who did not switch in the societal task, did switch in the individual task and could, thus, be included in the analysis of the DI-I. All analyses were also performed excluding the respondents who did not switch in the societal task, which did not yield different results. The results are available from the authors upon request). Another 9 subjects indirectly violated impatience by having $s_{i}>t_{i}$ for at least one indifference in DI-I, and 9 subjects (not necessarily the same) had $s_{i}>t_{i}$ in DI-S. These subjects were also dropped from the sample, although we could analyze the subparts including the subjects who did not violate impatience for one of the two tasks. Finally, two subjects had multiple switching points and were also removed from our sample. This resulted in 99-1-12-9-2 = 75 included subjects for the individual task, and 99-5-26-9-2 = 57 included subjects for the societal task. Figure 1 plots the distributions of the DI-indices for both tasks.

Table 1 classifies the subjects into increasing, decreasing, and constant impatience for each of the four choices (i.e., two for the individual task and two for the societal task). A comparison of DI-I-02 and DI-I-24 reveals evidence of quasihyperbolic discounting: DI-I-02 is significantly higher than DII-24 according to a Wilcoxon signed ranks test $(p<0.01)$. However, we did not find such a difference for the societal task $(p=0.81)$. Spearman rank correlation analyses showed a significant correlation between the two indices for both tasks $(p<0.01)$.

Table 2 shows summary measures of the DI-indices. These indicate a difference between the two tasks, with those of the individual task being higher than those of the societal task. Wilcoxon signed ranks tests confirm the significance of these differences at the $10 \%$ level ( $p<0.09$ for DI-02 and $p<0.02$ for DI-24). However, comparing the percentages of DI, CI, and II (as derived from Table 1), it turns out that such differences are not present for the discounting classifications ( $\chi^{2}$-tests, $\left.p>0.44\right)$. 

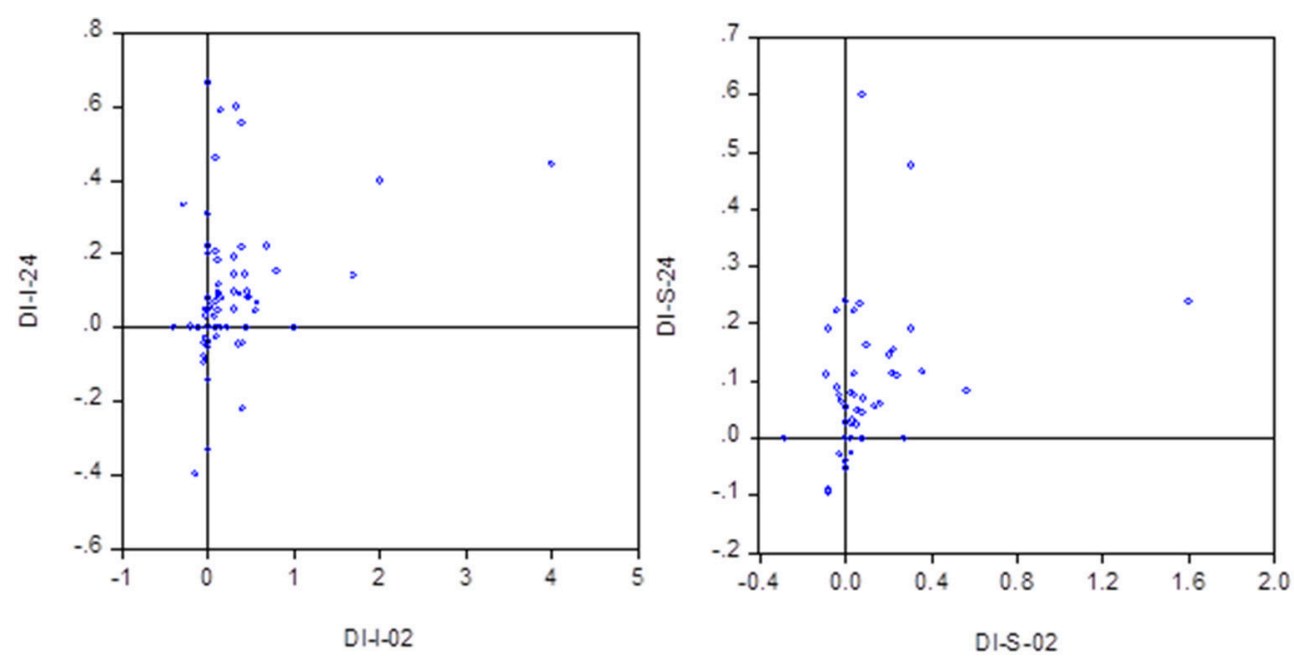

FIGURE 1 | Distributions individual DI indices (left) and societal DI-indices (right).

TABLE 1 | Classification of degree of impatience.

\begin{tabular}{|c|c|c|c|c|c|c|}
\hline & DI-I-02 & DI-I-24 & Total & DI-S-02 & DI-S-24 & Total \\
\hline Constant impatience & 21 (28\%) & 19 (25.3\%) & 40 (26.7\%) & $13(22.8 \%)$ & $13(22.8 \%)$ & 26 (22.8\%) \\
\hline Decreasing impatience & 42 (56\%) & 42 (56\%) & 84 (56\%) & $30(52.6 \%)$ & 34 (59.6\%) & $64(56.1 \%)$ \\
\hline Increasing impatience & 12 (16\%) & 14 (18.7\%) & $26(17.3 \%)$ & 14 (24.6\%) & 10 (17.5\%) & 24 (21.1\%) \\
\hline Total & 75 & 75 & 150 & 57 & 57 & 114 \\
\hline
\end{tabular}

\section{Discount Factors}

Because we used a qualitative health state improvement in the individual task, it was not possible to estimate discount factors for this task. For the societal task, on the other hand, this was possible, when assuming a linear societal utility function over number of patients. In the first choice list, the indifference under constant discounting is evaluated as in Equation (2), by:

$$
40=50^{*} \gamma^{t} \leftrightarrow \gamma=0.8^{1 / t}
$$

with $t$ in months and $\gamma$ the monthly discount factor. This resulted in average annual discount rates close to $30 \%$. However, it should be kept in mind that this is an overestimate of the true discount rate since the non-switchers are excluded. If we include them with the conservative assumption that these subjects have a discount rate of $0 \%$, the average discount rates reduce to rates around $10 \%$. We also performed the analysis assuming all these subjects would have switched at the next possible switching point, not included in the choice list. This gives their maximum possible discount rate (i.e., 5.68\%). The average discount rates are around $20 \%$ in that case. All these analyses did not show any differences between the three choice lists.

The social discount rates were not correlated with age or gender (Spearman test), but one of the behavioral variables was correlated with the discount rates. We found a negative correlation between living at home and the discount rate
( $p<0.02$ for all three discount factors). All other correlations were not significant.

\section{Relationship DI-Index With Demographics and Health Behavior}

Ninety-one subjects completed the survey. Table 3 provides summary statistics of the survey responses. None of the DIindices were correlated with age and gender, except for DI-S12 , which was correlated with gender $(p<0.05)$. In particular, women were found to have a lower DI-index than men. The regressions of each variable on the DI-indices also did not yield any significant coefficients, except for hours of sports, where the coefficient of DI-I-23 was positive and significant $(p=0.05)$. The direction of the latter correlation is counterintuitive.

\section{DISCUSSION}

This study has been the first to quantify the amount of time consistency for health outcomes, without distortions caused by utility curvature or the level of discounting. We find that the majority of subjects are decreasingly impatient for both individual and societal choices, but still there is a sizable minority with either constant or increasing impatience. Furthermore, the amount of decreasing impatience is higher for individual choices than for societal choices, although there is no such difference for the degree of decreasingly impatient choices. Hence, it seems 
TABLE 2 | Summary statistics DI-indices.

\begin{tabular}{lcccc}
\hline & DI-I-02 & DI-I-24 & DI-S-02 & DI-S-24 \\
\hline Mean (s.d.) & $0.24(0.57)$ & $0.08(0.19)$ & $0.08(0.24)$ & $0.07(0.13)$ \\
Median & 0.09 & 0.05 & 0.03 & 0.05 \\
Interquartile range & $0-0.35$ & $0-0.14$ & $0-0.08$ & $0-0.11$ \\
\hline
\end{tabular}

that those subjects who are decreasingly impatient, are so more strongly for individual choices than for societal choices, but the qualitative distribution of discount types does not differ between tasks. We also observe evidence in favor of quasi-hyperbolic discounting, with more decreasing impatience when the present is involved than when both outcomes occur in the future. Finally, we do not find many significant associations between the DIindices and the demographic and health-related data obtained from the survey.

Our results are largely similar to those of Rohde [1], indicating that both the frequency and the amount of decreasing impatience are similar for health and money. In particular, the data for both domains clearly reveal decreasing impatience, but at the same time highlight the necessity to also allow for agents with increasing impatience, which constitute a non-negligible part of the population. The results presented in this study also confirm the conclusion drawn by Bleichrodt et al. [4] with regard to the amount of decreasing and increasing impatience.

The reported study has a number of drawbacks. First, the experiment always started with the individual task. Future work could randomize these to control for order effects. A second drawback is the high amount of subjects for whom the DI-index could not be calculated, because they did not switch between the smaller-sooner outcome and the larger-later outcome. This problem may be addressed by extending the choice list, so that the DI-index can also be calculated for subjects with a low, but positive, time preference. Another possibility is to use a larger time unit, such as years instead of months. However, this will come at the expense of the precision of time preference estimates for early switchers. A third limitation is that, as in most time preference measurements, our design did not allow for negative discounting. Future work could therefore extend this study to allow for the assessment of negative discount rates, since there is some evidence for this, especially for outcomes framed as losses [27, 28], also in the health domain [29-31]. However, it is important to be aware that a violation of constant discounting then has a different interpretation, since time inconsistency for patient subjects means that they are either decreasingly or increasingly patient, instead of impatient. Moreover, the theoretical derivation of the DI-index [1] was only performed for the case of impatience, and, hence, it is not yet clear if the same results also apply to the case of patience. Fourth, our instructions told subjects to adopt chronic back pain as their neutral level of health. Because most subjects were healthy, chronic back pain could have been perceived as a loss and not as neutral. However, empirical evidence suggests that the reference point or neutral level of health can be manipulated and even healthy subjects usually adopt a health state which is worse than their current health if instructed to do so [32-35].
TABLE 3 | Summary statistics of demographic and behavioral variables.

\begin{tabular}{|c|c|c|}
\hline Variable & Description & Mean (SD) \\
\hline Gender & Male/female & $51.5 \%$ male \\
\hline Age & Age in years & $19.3(1.6)$ \\
\hline Health & Health on a 10-point Likert scale & $8.4(1.1)$ \\
\hline SLE & Subjective life expectancy & $84.1(9.9)$ \\
\hline Sports & $\begin{array}{l}\text { Number of hours of sports per } \\
\text { week }\end{array}$ & $4.5(2.9)$ \\
\hline Smoke & $\begin{array}{l}\text { Daily smoker/Smokes every now } \\
\text { and then/No-smoker }\end{array}$ & $\begin{array}{l}27.5 \% \text { smokes every } \\
\text { day or every now and } \\
\text { then }\end{array}$ \\
\hline Alcoholdays & $\begin{array}{l}\text { Average number of days drinking } \\
\text { alcohol per week }\end{array}$ & $1.6(1.1)$ \\
\hline Alcoholglasses & $\begin{array}{l}\text { Average number of glasses of } \\
\text { alcohol on drinking days }\end{array}$ & $4.5(3.9)$ \\
\hline Alcoholweek & $\begin{array}{l}\text { Average number of glasses of } \\
\text { alcohol per week } \\
\text { (AlcoholdaysAlcoholglasses) }\end{array}$ & $9.2(11.8)$ \\
\hline $\mathrm{BMl}$ & $\begin{array}{l}\text { Body Mass Index (Weight in kg } \\
\text { divided by length in meters } \\
\text { squared) }\end{array}$ & $21.6(2.2)$ \\
\hline Parents & $\begin{array}{l}\text { Dummy for subjects living with } \\
\text { their parents }\end{array}$ & $38 \%$ \\
\hline Nationality & Dummy for Dutch subjects & 62.6\% Dutch \\
\hline Saving & $\begin{array}{l}\text { Dummy for saving money (1) or } \\
\text { not (0) }\end{array}$ & $76 \%$ \\
\hline Monthly savings & Average monthly savings in euros & $€ 390.80(€ 2262.55)$ \\
\hline
\end{tabular}

Another future research avenue would be to extend the measurement of the DI-index to a more representative sample of the general public. This may also shed more light on the relationship between the amount of time inconsistency and health-related behaviors such as exercising, smoking, alcohol consumption, and body-mass index. That is, it may unravel if the lack of association in our study is the result of the low sample size or an inherent result, indicating that previously observed relations between time preference and healthy behavior are fully attributable to the level of impatience and risk aversion, instead of time inconsistency. Another explanation could be that this lack of association is related to our measurements, as we obtained estimates for health behavior through self-report, while DI-indices are obtained through revealed preference. Future work could attempt to test the association between DI and observed behaviors such as reallife exercise. Finally, future research can measure the DI-index for others' monetary outcomes (i.e., at the societal level for money).

Several implications arise from our study. First, health outcomes have similar characteristics as money with respect to degrees of time inconsistency. This holds both for the amounts of decreasing and increasing impatience, and for the amount of very patient choices. Second, the significant amount of increasing impatience implies that several common hyperbolic discount functions are not suitable to capture everyone's time preferences; hence, attention should be directed toward more general models such as the constant sensitivity model 
to accommodate increasing impatience for health outcomes $[20,36]$. Third, there is a difference between individual and societal time inconsistency in that the DI-index is higher for individual choices than for societal choices. However, the distribution of decreasingly, constantly, and increasingly impatient subjects does not differ between individual and societal health choices.

It can be concluded from our results that health and money show similar amounts of decreasing and increasing impatience. This highlights the need to look more deeply into discounting models that accommodate increasing impatience, instead of only focusing on the modeling of decreasing impatience. In addition, we have shown both similarities and discrepancies in time consistency between individual and societal tasks in the health domain. Finally, we did not find robust evidence of relations between the amount of time inconsistency and demographic characteristics or health-related behavior, although more research is needed to explore this relationship in more detail.

\section{REFERENCES}

1. Rohde KIM. Measuring decreasing and increasing impatience. Manage Sci. (in press).

2. Anderson S, Harrison G, Lau M, Rutstrom E. Eliciting risk and time preferences. Econometrica (2008) 76:583-618. doi: 10.1111/j.1468-0262.2008. 00848.x

3. Abdellaoui M, Attema AE, Bleichrodt H. Intertemporal tradeoffs for gains and losses: an experimental measurement of discounted utility. Econ J. (2010) 120:845-66. doi: 10.1111/j.1468-0297.2009.02308.x

4. Bleichrodt H, Gao Y, Rohde KIM. A measurement of decreasing impatience for health and money. J Risk Uncertain (2016) 52:213-31. doi: 10.1007/s11166-016-9240-0

5. Samuelson P. A note on the measurement of utility. Rev Econ Stud. (1937) 4:155-61. doi: 10.2307/2967612

6. Koopmans TC. Stationary ordinal utility and impatience. Econometrica (1960) 28:287-309. doi: 10.2307/1907722

7. Frederick S, Loewenstein G, O'Donoghue T. Time discounting and time preference: A critical review. J Econ Lit. (2002) 40:351-401. doi: $10.1257 /$ jel.40.2.351

8. O'Donoghue T, Rabin M. Doing it now or later. Am Econ Rev. (1999) 89:103-24. doi: 10.1257/aer.89.1.103

9. Phelps E, Pollak RA. On second-best national savings and game-equilibrium growth. Rev Econ Stud. (1968) 35:185-99. doi: 10.2307/2296547

10. Laibson D. Golden eggs and hyperbolic discounting. Q J Econ. (1997) 112:443-77. doi: 10.1162/003355397555253

11. Harvey CM. Value functions for infinite period planning. Manage Sci. (1986) 32:1123-39. doi: 10.1287/mnsc.32.9.1123

12. Herrnstein RJ. Self-control as response strength. In: Bradshaw CM, Szabadi E, Lowe CF. Quantification of Steady-State Operant Behavior. Amsterdam: Elsevier. (1981). p. 3-21.

13. Loewenstein G, Prelec D. Anomalies in intertemporal choice: evidence and an interpretation. Q J Econ. (1992) 107:573-97. doi: 10.2307/2118482

14. Takahashi T. Loss of self-control in intertemporal choice may be attributable to logarithmic time-perception. Med Hypotheses (2005) 65:691-3. doi: 10.1016/j.mehy.2005.04.040

15. Prelec D. Decreasing impatience: a criterion for non-stationary time preference and "hyperbolic" discounting. Scand J Econ. (2004) 106:511-32. doi: 10.1111/j.0347-0520.2004.00375.x

16. Frederick S, Loewenstein G, O’Donoghue T. Time discounting and time preference: a critical review. In: Loewenstein G, Read D, Baumeister R, editors.

\section{AUTHOR CONTRIBUTIONS}

AA designed the experiment. AA and SL jointly administered the experiment and analyzed the data. AA wrote a first draft of the manuscript, which was then complemented by SL. In the last phase of the study, AA and SL together finalized the submitted version of the manuscript.

\section{ACKNOWLEDGMENTS}

The authors gratefully acknowledge Salma Boulkhrif and Lindsey Meijer for their contribution to the data entry for this research project.

\section{SUPPLEMENTARY MATERIAL}

The Supplementary Material for this article can be found online at: https://www.frontiersin.org/articles/10.3389/fams. 2018.00016/full\#supplementary-material

Time and Decision: Economic and Psychological Perspectives on Intertemporal Choice. New York, NY: Russell Sage Foundation (2003). p. 13-86.

17. Attema AE. Developments in time preference and their implications for medical decision making. J Oper Res Soc. (2012) 63:1388-99. doi: 10.1057 /jors.2011.137

18. Bleichrodt H, Johannesson M. Time preference for health: a test of stationarity versus decreasing timing aversion. J Math Psychol. (2001) 45:265-82. doi: 10.1006/jmps.2000.1312

19. Attema AE, Bleichrodt H, Rohde KIM, Wakker PP. Time-tradeoff sequences for analyzing discounting and time inconsistency. Manage Sci. (2010) 56:2015-30. doi: 10.1287/mnsc.1100.1219

20. Bleichrodt $H$, Rohde KIM, Wakker PP. Non-hyperbolic time inconsistency. Games Econ Behav. (2009) 66:27-38. doi: 10.1016/j.geb.2008. 05.007

21. Rohde KIM. The hyperbolic factor: a measure of time inconsistency. J Risk Uncertain (2010) 41:125-40. doi: 10.1007/s11166-010-9100-2

22. Hardisty DJ, Weber EU. Discounting future green: money versus the environment. J Exp Psychol Gen. (2009) 138:329-40. doi: 10.1037/a00 16433

23. Van der Pol M, Cairns J. Descriptive validity of alternative intertemporal models for health outcomes: an axiomatic test. Health Econ. (2011) 20:770-82. doi: 10.1002/hec.1628

24. Holt CA, Laury SK. Risk aversion and incentive effects. Am Econ Rev. (2002) 92:1644-55. doi: 10.1257/000282802762024700

25. Ameriks J, Caplin A, Leahy J, Tyler T. Measuring self-control problems. Am Econ Rev. (2007) 97:966-72. doi: 10.1257/aer.97.3.966

26. Strathman A, Gleicher F, Boninger DS, Edwards CS. The consideration of future consequences. J Pers Soc Psychol. (1994) 66:742-52. doi: 10.1037/0022-3514.66.4.742

27. Kapteyn A, Teppa F. Hypothetical intertemporal consumption choices. Econ J. (2003) 113:140-52. doi: 10.1111/1468-0297.00111

28. Barsky RB, Juster FT, Kimball MS, Shapiro MD. Preference parameters and behavioral heterogeneity: An experimental approach in the health and retirement study. Q J Econ. (1997) 112:537-79. doi: 10.1162/0033553975 55280

29. Chapman GB, Coups EJ. Time preferences and preventive health behavior: acceptance of the influenza vaccine. Med Decison Making (1999) 19:307-14. doi: 10.1177/0272989X9901900309

30. der Pol MM, Cairns JA. Negative and zero time preference for health. Health Econ. (2000) 9:171-5. doi: 10.1002/(SICI)1099-1050(200003)9:2<171::AIDHEC492>3.0.CO;2-Z 
31. Ganiats TG, Carson RT, Hamm RM, Cantor SB, Sumner W, Spann SJ. Population-based Time preferences for future health outcomes. Med Decison Making (2000) 20:263-70. doi: 10.1177/0272989X00020 00302

32. Bleichrodt H, Pinto JL. Loss aversion and scale compatibility in twoattribute trade-offs. J Math Psychol. (2002) 46:315-37. doi: 10.1006/jmps.2001. 1390

33. Attema AE, Brouwer WBF, l'Haridon O. Prospect theory in the health domain: a quantitative assessment. J Health Econ. (2013) 32:1057-65. doi: 10.1016/j.jhealeco.2013.08.006

34. van Osch SMC, van den Hout WB, Stiggelbout AM. Exploring the reference point in prospect theory: gambles for length of life. Med Decison Making (2006) 26:338-46. doi: 10.1177/0272989X062 90484

35. Lipman SA, Attema AE, Brouwer WBF. QALYs Without Bias? Non-Parametric Correction of Time Trade-off And Standard Gamble Utilities Based on Prospect
Theory. Rotterdam: SSRN Working paper. (2017). doi: 10.2139/ssrn.30 51140

36. Ebert JEJ, Prelec D. The fragility of time: time-insensitivity and valuation of the near and far future. Manage Sci. (2007) 53:1423-38. doi: $10.1287 / \mathrm{mnsc} .1060 .0671$

Conflict of Interest Statement: The authors declare that the research was conducted in the absence of any commercial or financial relationships that could be construed as a potential conflict of interest.

Copyright (c) 2018 Attema and Lipman. This is an open-access article distributed under the terms of the Creative Commons Attribution License (CC BY). The use, distribution or reproduction in other forums is permitted, provided the original author(s) and the copyright owner are credited and that the original publication in this journal is cited, in accordance with accepted academic practice. No use, distribution or reproduction is permitted which does not comply with these terms. 